Pacific Journal of Mathematics

SLENDER RINGS AND MODULES 


\title{
SLENDER RINGS AND MODULES
}

\author{
E. L. LADY
}

An abelian group $B$ is called slender if every homomorphism from $\Pi_{1}^{\infty} Z$ into $B$ is continuous for the discrete topology on $B$ and the topology that $\Pi_{1}^{\infty} Z$ has as a cartesian product if $Z$ (the additive group of integers) is considered discrete. It can be seen that if $B$ is slender, then every homomorphism from $\Pi_{1}^{\infty} A_{i}$ into $B$ is continuous for the same topologies, where $A_{i}$ are arbitrary abelian groups. Slender groups were completely characterized by Nunke, and it follows from his characterization that all countable reduced torsion free groups are slender. Nunke's results depend on the fact that $Z$ is slender (proved by Specker) and the use of some homological machinery. In this paper, slender modules are studied over an arbitrary ring. A ring $R$ will be called slender if $R$ is a slender $R$-module. In Theorem 1 , a generalization of the Baire Category Theorem is used to show that a countable module over an arbitrary ring is slender if $\cap \mathfrak{m} B=0$, where $\mathfrak{m}$ ranges over the set of ideals in $R$ which are not zero divisors for $B$. It follows that countable torsion free reduced modules over any (countable) integral domain are slender. In Theorem 2 , it is shown that a commutative ring $R$ is slender if there exists an infinite set of maximal ideals with the property that the intersection of any infinite subset is 0 .

In $\S 2$, reflexive modules over a slender ring are studied, and it is shown that a projective module is reflexive if it is generated by a set with cardinality smaller than the first measurable cardinal. In $\S 3$, Nunke's characterization is extended to modules over a slender Dedekind domain having only countably many ideals. The general approach in $\S 3$ follows Nunke's, but all homological machinery is avoided by the use of topological techniques.

$R$ will always be a ring with identity. The word ideal will mean a two sided ideal, the word module will mean a unitary left module.

If $B$ is an $R$-module and $\mathfrak{m}$ an ideal in $R$, we say that $\mathfrak{m}$ is a zero divisor for $B$ if $m x=0$ for some nonzero $x \in B$. We say that $B$ is torsion free if no nonzero ideal is a zero divisor for $B$. If $\mathscr{C}$ is a family of ideals in $R$, then by the $\mathscr{C}$-adic topology on $B$ we mean the linear topology induced by the family of submodules $\mathrm{m} B$ where $\mathfrak{m}$ is a finite intersection of ideals in $\mathscr{C l}$. The word complete will only be used in reference to Hausdorff topologies. 
1. Slender modules. Let $\left\{A_{i} \mid i \in I\right\}$ be a family of $R$ modules. By the product topology on $\Pi_{I} A_{i}$ we mean the topology this module has as a Cartesian product if each $A_{i}$ is considered discrete. In the product topology, $\Pi_{I} A_{i}$ is a complete topological module. If $a \in \Pi_{I} A_{i}$, we will write $a(i)$ to denote the $i$ th coordinate of $a$. If $J \subseteq I$, we will consider $\Pi_{J} A_{i}$ as a submodule of $\Pi_{I} A_{i}$ in the usual way.

If $\mathscr{C l}$ is a family of ideals in $R$, we will also be interested in the topology on $\Pi_{r} A_{i}$ which is the supremum of the product topology and the $/ \mathscr{C}$-adic topology. We will refer to this as the strong (or $\mathscr{C l}$-strong) topology. It is a complete topology, and is metrizable if both $\mathscr{C l}$ and $I$ are countable. If $a \in \Pi_{I} A_{i}$, then the sets of the form $a+\mathfrak{m} \prod_{i \notin J} A_{i}$ form a basis for the neighborhood system at $a$ in the strong topology, where $J$ is a finite subset of $I$ and $m$ is a finite intersection of ideals in $\mathscr{C}$. Since these neighborhoods are closed in the product topology, the following generalization of the Baire Category Theorem is applicable.

LeMma 1. Let $X$ be a complete metric space. Let $\mathscr{T}$ be a topology on $X$ which is finer than the metric topology and such that $\mathscr{T}$ has a basis consisting of sets which are closed in the metric topology. Then $X$ is not a countable union of sets which are nowhere dense with respect to $\mathscr{T}$.

Proof. Let $G_{1}, G_{2}, \cdots$ be a countable family of sets which, with respect to $\mathscr{T}$, are dense and open. Then we can inductively find a sequence of points $x_{n}$ and $\mathscr{T}$-neighborhoods $U_{n}$ of $x_{n}$ such that, for each $n, U_{n+1} \subseteq U_{n} \cap G_{1} \cap \cdots \cap G_{n+1}$, and such that the $U_{n}$ are closed in the metric topology and have diameter less than $1 / n$. It follows that $\left\{x_{n}\right\}$ is a Cauchy sequence in the metric topology and hence converges metrically to a point $x$. Since the $U_{n}$ are metrically closed, it follows that $x \in \cap G_{n}$. Thus any countable intersection of dense open sets is nonempty, and this is equivalent to the assertion of the lemma.

Corollary 1. If $\mathscr{t}$ is a family of ideals, then $\Pi_{1}^{\infty} A_{i}$ is not a countable union of sets which are nowhere dense for the strong topology.

We are now able to prove the two main theorems.

Definition. If $B$ is an $R$-module, we say that $B$ is (left) slender if for every countable family of $R$-modules $\left\{A_{i}\right\}$ and every homomorphism $\phi$ from $\Pi_{1}^{\infty} A_{i}$ into $B$, there is a natural number $n$ such that $\phi\left(\prod_{i \geqq n} A_{i}\right)=0$. This amounts to saying that $\phi$ is continuous 
for the product topology on $\prod_{1}^{\infty} A_{i}$ and the discrete topology on $B$. We say that $R$ is a (left) slender ring if $R$ is a slender $R$-module. (The concept of right slenderness can, of course, be similarly defined.)

Theorem 1. Let $B$ be a countable $R$-module and $\mathscr{l l}$ be the set of all ideals which are not zero divisors for $B$. If the Al-adic topology on $B$ is Hausdorff, then $B$ is slender.

Proof. Let $\phi$ be a homomorphism from $\Pi_{1}^{\infty} A_{i}$ into $B$. Then $\Pi_{1}^{\infty} A_{i}$ is a countable union of sets $\phi^{-1}(x)$, for $x \in B$. Because $\phi$ is necessarily continuous in the $\mathscr{C}$-adic topology, each $\phi^{-1}(x)$ is closed in the $\mathscr{C}$-adic topology, and hence in the strong topology. By Corollary 1, some $\phi^{-1}(x)$ must have an interior in the strong topology. Thus there is a natural number $n$ and an ideal $m \in \mathscr{C}$ such that $\phi\left(a+\mathfrak{m} \prod_{i \geq n} A_{i}\right)=\{x\}$ for some $a \in \Pi A_{i}$. This is only possible if $\phi\left(\mathfrak{m} \prod_{i \geqq n} A_{i}\right)=0$. Since $\mathfrak{m}$ is not a zero divisor for $B$, this gives $\phi\left(\prod_{i \geqq n} A_{i}\right)=0$.

EXAMPLE 1. Let $R$ be a countable commutative integral domain which is not a field. The $R$ is a slender ring, and every countable reduced torsion free $R$-module $B$ is slender. (Reduced means that no submodule is divisible.) In fact, in both cases $\mathscr{C}$ is the set of all nontrivial ideals in $R$. If $m \in \mathscr{l l}$ and $0 \neq x \in R$, then $x \notin \mathrm{m} x$ and $\mathrm{m} x \in \mathscr{C}$, so $R$ is $\mathscr{C l}$-adically Hausdorff. If $B$ is torsion free, then it is easily seen that $\bigcap_{m} \mathfrak{m} B$ is divisible and hence equal to 0 if $B$ is reduced.

ExAmple 2. If $R$ is a countable Dedekind domain, then the hypotheses of Theorem 1 hold if and only if $B$ is countable torsion free and reduced. In fact, clearly it is necessary that $B$ be reduced. Now if $B$ is not torsion free, then there is a submodule $A$ of $B$ with $\mathfrak{a}=\operatorname{Ann}(A) \neq 0$. Now every ideal in $R$ is a product of maximal ideals. If $\mathfrak{m}$ is maximal and $a \subseteq \mathfrak{m}$, them $\mathfrak{m}$ is an associated prime for $A$, so $m \notin \mathscr{K}$. On the other hand, if $m$ does not contain $a$, then $\mathfrak{m}+\mathfrak{a}=R$ so that $\mathfrak{m} A=A$. Hence we have $A=\bigcap_{m} \mathfrak{m} A \subseteq \bigcap_{m} \mathfrak{m} B$.

EXAMPLE 3. In general, $B$ need not be torsion free in order to satisfy the hypothesis for Theorem 1. For instance, let $R=Z[x]$ and $B=R / 2 R$. Then $B$ is not a torsion free $R$-module, but the hypothesis of Theorem 1 is easily seen to hold.

We cannot hope that Theorem 1 will remain valid if we simply require that $B$ be countably generated. For instance, if $R$ is a complete local ring, then $R$ fails to be slender, as can be seen from 
the proof of Theorem 6. However, there is some hope for certain uncountable rings, as is shown in Theorem 2 .

THEOREM 2. Let $R$ be a commutative ring having an infinite set of maximal ideals with the property that the intersection of any infinite subset of $\mathscr{C}$ is 0 . Then $R$ is a slender ring.

Proof. $R$ must be an integral domain, since if $x y=0$, then either $x$ or $y$ must belong to infinitely many ideals in $\mathscr{C}$ (since these are prime). There is no loss of generality in assuming that $\mathscr{C}$ is countable, so we may enumerate $\mathscr{C l}$ as $\left\{\mathfrak{m}_{i j} \mid i, j=1,2, \cdots\right\}$. Now let $C_{m n}=\mathbf{U}_{i=1}^{n}\left(R \backslash \mathrm{m}_{m i}\right)$ for every pair of natural numbers $m, n$. Let $C_{0}=\{0\}$ and for $n \geqq 1$ let $C_{n}=\bigcap_{m} C_{m n}$. Then $C_{n}$ is $\mathscr{C}$-adically closed and if $0 \neq x \in R$, then since $x$ can belong to only finitely many $\mathfrak{m}_{i j}, x \in \bigcup_{n} C_{n}$. Now if $a$ is a nonzero ideal in $R$, then there are only finitely many $\mathfrak{m}_{i j}$ with $a \subseteq \mathfrak{m}_{i j}$. Thus for some $m$, none of the ideals $\mathfrak{m}_{m j}$ contain $a$. Thus for any fixed $b \in R$ and every $n$, we can find $x \equiv b(\bmod \Omega)$ with $x \in \mathfrak{n t}_{m i}, i=1, \cdots, n$ by the Chinese remainder theorem. Hence $b+a$ is not contained in $C_{m n}$ for any $n$, and so is not contained in any $C_{n}$.

Now let $\phi$ be a homomorphism from $\Pi_{1}^{\infty} A_{i}$ into $R$. It now follows by Corollary 1 that some $\phi^{-1}\left(C_{n}\right)$ has an interior in the strong topology. This means that $\phi\left(a+\mathfrak{m} \prod_{i \geqq m} A_{i}\right) \subseteq C_{n}$ where $a \in \prod A_{i}, m$ is a natural number, and $\mathfrak{n}$ is a finite intersection of ideals in $\mathscr{C}$. Since $\phi\left(\mathfrak{m} \prod_{i \geqq m} A_{i}\right)$ is an ideal in $R$, it follows from what we have said about the $C_{n}$ that this can only happen if $\mathfrak{m} \phi\left(\Pi_{i \geqq m} A_{i}\right)=0$, and hence $\phi\left(\prod_{i \geqq m} A_{i}\right)=0$, since $R$ is an integral domain.

Example $4 . \quad R$ is a Dedekind domain with infinitely many prime ideals.

Example 5. $R=K\left[x_{1}, \cdots, x_{s}\right]$ where $K$ is a field and the $\mathrm{x}_{i}$ are indeterminates. Let $F$ be the algebraic closure of $K$ and $V=$ $F \otimes R$, regarded as an $F$-vector space. Let $V_{n}$ be the subspace of $V$ generated by those polynomials of degree at most $n$, let $r(n)=$ $\operatorname{dim} V_{n}$, and let $V_{n}^{1}$ be the kernel of the restriction map $V^{*} \rightarrow V_{n}^{*}$, where $V^{*}=\operatorname{Hom}(V, F)$. Each point $a \in F^{s}$ induces a linear functional $\phi(a) \in V^{*}$ by $\phi(a)(f)=f(a)$. Furthermore, suppose $W_{1}, \cdots, W_{r}$ are proper subspaces of $V^{*}$ containing $V_{n}^{1}$. Choose $f_{i} \in V_{n}$ for each $1 \leqq i \leqq r$ such that $f_{i}$ is annihilated by $W_{i}$. Now there is a point $a \in F^{s}$ such that $f_{1} \cdots f_{r}$ does not vanish at $a$, hence we see that $\phi\left(F^{s}\right) \nsubseteq W_{1} \cup \cdots \cup W_{r}$. This enables us to inductively choose a sequence of points $\left\{a_{n}\right\}$ as follows: choose $a_{n}$ such that for all $i \leqq n$ and all $X \leqq\left\{a_{1}, \cdots, a_{n-1}\right\}$, if $\left\langle V_{i}^{1}, \phi(X)\right\rangle \neq V^{*}$, then $\phi\left(a_{n}\right) \notin\left\langle V_{i}^{1}, \phi(X)\right\rangle$. 
It can now be seen that no nonzero polynomial of degree $n$ vanishes at any $r(n)+n$ of these points. Now for each $a_{n}$, we have the maximal ideal in $R$ consisting of all polynomials vanishing at $a_{n}$. Let $\mathscr{C}$ be this set of maximal ideals.

Example 6. A similar technique will work for $R=K\left[x_{1}, \cdots\right.$, $\left.x_{s}\right] / \mathfrak{p}$, where $\mathfrak{p}$ is a nonmaximal prime ideal.

The following theorem gives some properties of slender modules which are well known in abelian group theory [3, Theorems 47.2 and 47.4].

THEOREM 3. (1) Submodules of slender modules are slender.

(2) A direct sum of slender modules is slender.

(3) $B$ is slender if and only if every homomorphism of from $\Pi_{1}^{\infty} R$ into $B$ is continuous for the product topology and discrete topology respectively.

(4) If $B$ is slender and $I$ is a set whose cardinality is smaller than the first measurable cardinal, then every homomorphism from $\Pi_{I} A_{i}$ to $B$ is continuous for the product topology on $\Pi_{I} A_{i}$ topology on $B$.

\section{Proof. (1) Obvious.}

(2) Let $\phi: \Pi_{1}^{\infty} A_{i} \rightarrow \sum_{K} B_{k}$ where each $B_{k}$ is slender. Let $\phi_{k}$ be the composition of $\phi$ with the projection onto $B_{k}$. Since $B_{k}$ is slender, each $\phi_{k}$ is continuous for the product and discrete topologies. We must show that if $\left\{a_{n}\right\}$ is a sequence of elements in $\Pi A_{i}$ which converges to 0 in the product topology, then $\phi\left(a_{n}\right)$ is eventually 0 . Since there are in any case only countably many $k$ for which $\phi_{k}\left(a_{n}\right) \neq$ 0 for some $n$, we may suppose that we are dealing with $\sum_{1}^{\infty} B_{k}$. Now for any $n, \dot{\phi}^{-1}\left(\sum_{1}^{n} B_{k}\right)=\bigcap_{k>n} \phi_{k}^{-1}(0)$ which is closed in the product topology by the continuity of each $\phi_{k}$. Since $\Pi A_{i}=$ $\bigcup_{n=1}^{\infty} \phi^{-1}\left(\sum_{1}^{n} B_{k_{k}}\right)$, the Baire Category Theorem implies $\phi\left(\prod_{i \geqq m} A_{i}\right) \subseteq$ $\sum_{1}^{n} B_{k}$ for some $m$ and $n$. Since $\sum_{1}^{n} B_{k}$ is clearly slender, the result follows.

(3) Let $\phi: \Pi_{1}^{\infty} A_{i} \rightarrow B$, and let $\left\{a_{n}\right\}$ be a sequence of elements in $\prod_{1}^{\infty} A_{i}$ converging to 0 in the product topology. For every natural $n$, let $e_{n} \in \Pi_{1}^{\infty} R$ be defined by $e_{n}(i)=1$ if $i=n, e_{n}(i)=0$ otherwise. We can define a homomorphism $f: \Pi_{1}^{\infty} R \rightarrow \Pi_{1}^{\infty} A_{i}$ such that $f\left(e_{n}\right)=$ $a_{n}$. Now if the composition $\phi f: \prod_{1}^{\infty} R \rightarrow B$ is continuous, then $\phi\left(a_{n}\right)=$ of $f\left(e_{n}\right)$ is eventually 0.

(4) (Due to Los). Let $\phi: \Pi_{I} A_{i} \rightarrow B$ where $I$ has nonmeasurable cardinality and $B$ is slender. It is clear that if there are infinitely many $A_{i}$ with $\phi\left(A_{i}\right) \neq 0$, then we get a contradiction to 
the fact that $B$ is slender by taking a countably infinite subset of these and considering the appropriate restriction of $\phi$. Hence we see that it suffices to show that if $\phi\left(\sum_{I} A_{i}\right)=0$, then $\phi\left(\Pi_{I} A_{i}\right)=0$. Suppose this is not the case, and choose $a \in \Pi_{I} A_{i}$ with $\phi(a) \neq 0$. Now for each subset $X$ of $I$, let $a_{X} \in \Pi_{I} A_{i}$ be defined by $a_{X}(i)=$ $a(i)$ if $i \in X, a_{X}(i)=0$ otherwise. We see that the family of sets $X$ for which $\phi\left(a_{X}\right)=0$ is a $\sigma$-algebra $S$ which contains all the finite subsets of $I$. It also follows from the fact that $B$ is slender that if we have a family of disjoint subsets $X_{i}$ of $I$, we can have $\phi\left(a_{X_{i}}\right) \neq$ 0 for at most finitely many $X_{i}$. From this we see that the quotient algebra $P / S$ is finite, where $P$ is the $\sigma$-algebra of all subsets of $I$. By choosing an atom of $P / S$, we can define a nontrivial measure on $P$ which is countably additive and takes only 0 and 1 as values, and is 0 on all finite sets. This contradicts the fact that $I$ has nonmeasurable cardinality.

REMARKs. (1) The first measurable cardinal is at least as large as the first inaccessible cardinal [7].

(2) The nonmeasurability of $|I|$ is necessary, as is shown by the following example. Let $I$ be a measurable cardinal and consider $\Pi_{I} Z$. Let there be given a 0,1 -valued measure on $I$ which is 0 on finite sets. For each $a \in \Pi_{I} Z$, there is exactly one integer $n$ such that the set $\{i \in I \mid a(i)=n\}$ has measure 1. Define $\phi(a)=n$. Then it is easily seen that $\phi$ is a homomorphism from $\Pi_{I} Z$ to $Z$ which is not continuous for the product and discrete topologies.

COROLlaRY 2. $R$ is slender if and only if every projective $R$-module is slender. In particular, the slenderness of $R$ can be regarded as a property of the category of $R$ modules.

CoROllary 3. If $R$ is a subring of $S$ such that $S$ is a slender $R$-module, then $S$ is a slender ring. In particular, this is true if $R$ is a slender ring and $S$ a projective $R$-module.

Proof. This follows because any homomorphism of $S$-modules can be regarded as a homomorphism of $R$-modules.

COROLlaRy 4. If $R$ is a slender ring, then so is the polynomial ring $R[x]$, the ring of $n$ dimensional matrices over $R$, and the group ring $R[G]$, for any group $G$. In addition, if $R$ is an algebra over a field $K$ and $F$ is an extension field of $K$, then $F \otimes_{K} R$ is slender.

Proof. In each case, the larger ring is a free $R$-module, so this follows from Corollary 3. 
2. Reflexive modules. If $A$ is an $A$-module, we write $A^{*}=$ Hom $(A, R) . \quad\left(A^{*}\right.$ is a right $R$-module.) We say that $A$ is reflexive if the natural map $\psi: A \rightarrow A^{* *}$ given by $\psi(a)(f)=f(a)$ is an isomorphism.

Theorem 4. Let $R$ be a ring and $\left\{M_{i} \mid i \in I\right\}$ a family of $R$ modules, with $|I|$ nonmeasurable. If $R$ is slender, then $\left(\Pi_{I} M_{i}\right)^{*} \approx$ $\sum_{I} M_{i}$. If the $M_{i}$ are reflexive, then $\Pi_{I} M_{i}$ is reflexive if $R$ is slender, and $\sum_{I} M_{i}$ is reflexive if $R$ is right slender.

Proof. The fact that $\left(\Pi_{I} M_{i}\right)^{*} \approx \sum_{I} M_{i}^{*}$ when $R$ is slender follows from Theorem 3,4. Since we have $\left(\sum_{I} M_{i}\right)^{*} \approx \Pi_{I} M_{i}^{*}$ in any case, the other assertions follow immediately.

COROLlaRy 5. If $R$ is a right slender ring, then every projective module generated by a set with nonmeasurable cardinality is reflexive.

Proof. Since summands of reflexive modules are reflexive, it suffices to prove the corollary for free modules, but this is immediate from the theorem and the fact that $R$ is reflexive as an $R$-module.

CoROllaRY 6. Let $R$ be a slender ring such that every nonfinitely generated projective right $R$-module is free. Let $|I|$ be nonmeasurable and $\Pi_{I} R=A \oplus B$. Then either $A$ is a finitely generated projective module or $A \approx \Pi_{K} R$ with $K \subseteq I$.

Proof. Since summands of reflexive modules are reflexive, $A^{* *} \approx A$ by Theorem 4 . We have $\left(\Pi_{I} R\right)^{*} \approx A^{*} \oplus B^{*}$, so $A^{*}$ is projective since $\left(\Pi_{I} R\right)^{*}$ is free. If $A^{*}$ is finitely generated, then $A^{* *}$ is a finitely generated projective module. Otherwise, $A^{*} \approx$ $\sum_{K} R^{*}$ and $A^{* *} \approx \Pi_{K} R$.

3. Dedekind domains. In this section, $R$ will be a Dedekind domain and $\mathscr{M}$ will always be the set of nonzero ideals in $R$. When $R$ is slender and $\mathscr{C}$ is countable, we will be able to completely characterize slender $R$-modules, as was done by Nunke [5] and [6], for the case $R=Z$. We will use some topological observations to avoid the homological machinery involved in Nunke's proof.

Throughout this section, we will write $P=\Pi_{1}^{\infty} R_{i}$, with $R_{i}=R$. We will write $U_{n}=\prod_{i \geqq n} R_{i}$ (considered as a submodule of $P$ ), so that the $U_{n}$ form a neighborhood basis at 0 for the product topology on $P$. The supremum of the $\mathscr{C l}$-adic and product topologies on $P$ will again be called the strong topology. We recall that $P$ is complete in the strong topology. 
Theorem 5. Let $R$ be a Dedekind domain and $P=\Pi_{1}^{\infty} R_{i}, R_{i}=$ $R$. If $A$ is a submodule of $P$ which is closed in the product topology, then $A \approx \Pi N_{i}$, where each $N_{i}$ is a projective module. In particular, if rank $A$ is finite, then $A$ is projective. If $R$ is slender and $A$ has infinite rank, then $A \approx P$.

Proof. For each $n$, we let $V_{n}=A \cap U_{n}$ and we let $\pi_{n}$ be the projection of $P$ onto $R_{n}$. Then the restriction of $\pi_{n}$ to $V_{n}$ has kernel $V_{n+1}$ and $\pi_{n}\left(V_{n}\right)$ is projective (as a submodule of $\left.R_{n}\right)$. Hence we have $V_{n}=N_{n} \oplus V_{n+1}$ where $N_{n}$ is projective, i.e., we get $A=N_{1} \oplus$ $N_{2} \oplus \cdots \oplus N_{n} \oplus V_{n+1}$. It is readily seen that this gives us an isomorphism from $A$ to $\Pi N_{i}$. Now if there are only finitely many nonzero $N_{i}$, then $A$ is finite rank projective. Otherwise, if $R$ is slender, we have $A \approx\left(\Pi N_{i}\right)^{* *} \approx\left(\sum N_{i}^{*}\right)^{*} \approx\left(\sum R\right)^{*} \approx \Pi R$, because nonfinitely generated projective modules are free.

COROLlaRY 7. If $R$ is slender and $A$ is a countably generated module, then $A^{*}$ is reflexive and $A^{* *}$ is projective.

Proof. $A^{*}$ is submodule of $\Pi_{K} R$ which is closed in the product topology, where $K$ is a set of generators for $A$. The result now follows from Theorems 4 and 5 .

We now proceed ta a sequence of lemmas leading directly to the characterization of slender modules.

Lemma 2. Let $A$ be an $R$-module with a metrizable topology. If $A$ is complete and $B$ a closed submodule, then the quotient topology on $A / B$ is complete.

Proof. [2, §3, Prop. 4].

COROLlary 8. If $\mathscr{t h}$ is countable and $\left\{A_{i} \mid i=1,2, \cdots\right\}$ is any countable family of $R$ modules, then the reduced part of $\Pi A_{i} / \sum A_{i}$ is $\mathscr{C l}$-adically complete.

Proof. $\Pi A_{i}$ is complete in the strong topology. The $\mathscr{C}$-adic closure $K$ of $\sum A_{i}$ in $A_{i}$ consists of those $x$ such that, for every $\mathfrak{n} \in \mathscr{M}, x(i) \in \mathfrak{m} A_{i}$ for sufficiently large $i$. It is evident that this is also the closure of $\sum A_{i}$ in the strong topology and that $K / \sum A_{i}$ is divisible. Hence $\Pi A_{i} / K$ is the reduced part of $\Pi A_{i} / \sum A_{i}$. Also, because $K$ is dense in $\Pi A_{i}$ in the product topology, the topology induced on $\Pi A_{i} / K$ by the strong topology on $\Pi A_{i}$ is the same as the $\mathscr{C}$-adic topology on this module. Hence the result follows from Lemma 2. 
Lemma 3. If $\mathscr{C}$ is countable and $A$ is a submodule of $P$ such that $P / A$ is torsion free and $(P / A)^{*}=0$, then the reduced part of $P / A$ is /l-adically complete.

Proof. Because $P / A$ is torsion free, the reduced part of $P / A$ is

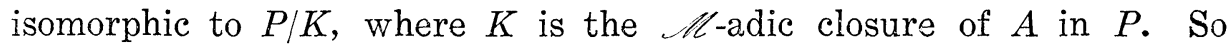
without loss of generality, we may assume that $A$ is $\mathscr{C}$-adically closed. Now $P /\left(A+U_{n}\right)$ is a finitely generated quotient of $P / A$. If this is not a torsion module, then it has a projective summand and it follows that $(P / A)^{*} \neq 0$. Hence for each $n$ there is a nonzero ideal $\mathfrak{m}_{n}$ such that $\mathfrak{m}_{n} P \subseteq A+U_{n}$. This means that the $/ \mathbb{l}$-adic topology on $P / A$ is also the quotient topology that $P / A$ inherits when $P$ is given the strong topology. Thus the result follows from Lemma 2.

REMARK. Corollary 8 and Lemma 3 fail if $\mathscr{t}$ is uncountable. In fact, let $A$ be the $\mathscr{C}$-adic closure in $P$ of $\sum_{1}^{\infty} R$. If $R$ is slender, then clearly $(P / A)^{*}=0$. Now choose $a \in P \backslash A$, and let $\mathfrak{n n}_{0}$ be a maximal ideal. For each set of ideals $\mathfrak{m}_{0}^{k_{0}}, \cdots, \mathfrak{m}_{n}^{k_{n}}$, where $\mathfrak{m}_{1}, \cdots, \mathfrak{m}_{n}$ are maximal ideals distinct from $\mathfrak{m}_{0}$, consider the set of $x$ such that $\therefore \equiv a\left(\bmod \mathfrak{m}_{0}^{k_{0}}\right)$ and $x \in \mathfrak{m}_{i}^{k_{i}}, i=1, \cdots, n$. These sets form a $\mathscr{C l}$ adically Cauchy filter in $P / A$, and hence, if $P / A$ is complete, converge to a point $x$ such that $x \neq 0$, and $x \in \mathfrak{m}$ for every maximal ideal $\mathfrak{m}$ distinct from $\mathfrak{m}_{0}$. Then if $y$ is a representative in $P$ for $x$, we have $y \notin A$, and every maximal ideal $\mathfrak{m} \neq \mathfrak{n}_{0}$, almost all the coordinates of $y$ belong to 11 . If $\mathscr{C}$ is uncountable, this implies that almost all the coordinates of $y$ must be 0 , which is a contradiction.

LemMA 4. Let $R$ be slender and $A \subseteq P$, and let $A^{\prime \prime}$ be the set of $x \in P$ such that $f(x)=0$ for every $f \in P^{*}$ for which $f(A)=0$. Then $A^{\prime \prime}$ is a summand of $P$ and $\left(A^{\prime \prime} / A\right)^{*}=0$.

Proof. We follow Nunke's proof. The fact that $\left(A^{\prime \prime} / A\right)^{*}=0$ follows easily once we know that $A^{\prime \prime}$ is a summand. Let $B$ be the image of the natural map $P^{*} \rightarrow A^{*}$. By Theorem $4, P^{*}$ is countably generated, hence $B$ is countably generated. Now $B \cong A^{*} \subseteq \Pi_{A} R$. Just as for abelian groups [3, Theorem 47.1], it is readily shown that these facts imply that $B$ is projective. Thus the sequence

$$
0 \longrightarrow(P / A)^{*} \longrightarrow P^{*} \longrightarrow B \longrightarrow 0
$$

is split exact. Dualizing, we get a split exact sequence

$$
0 \longrightarrow B^{*} \longrightarrow P \longrightarrow(P / A)^{* *} \longrightarrow 0 \text {. }
$$

But it is easily seen that the image of $B^{*}$ in $P$ is exactly $A^{\prime \prime}$. 
Lemma 5. Let $R$ be slender and $\mathscr{A}$ countable. If a torsion free module $B$ is a homomorphic image of $P$, then the reduced part of $B$ is the direct sum of a $\mathscr{C l}$-adically complete module and a module which is either finite rank projective or isomorphic to $P$.

Proof. Let $A$ be the kernel of $\phi: P \rightarrow B$. We have $P=A^{\prime \prime} \oplus C$ (Lemma 4) where $C$ is either finite rank projective or isomorphic to $P$ (Corollary 6). Hence $B \approx\left(A^{\prime \prime} \mid A\right) \oplus C$. Since $\left(A^{\prime \prime} \mid A\right)^{*}=0$, the result follows from Lemma 3 .

THEOREM 6. Let $R$ be a slender Dedekind domain such that the set $\mathscr{C}$ of nonzero ideals in $R$ is countable. Then an $R$-module $B$ is slender if and only if $B$ is reduced, torsion free, and contains no subgroup which is isomorphic to $P$ or $\mathscr{C l}$-adically complete.

Proof. Sufficiency. By Theorem 3, it suffices to show that if $\phi: P \rightarrow B$, then $\phi$ is continuous for the product and discrete topologies. From Lemma 5 and the hypothesis, the image of $\phi$ must be finite rank projective and hence, by Corollary 2, slender, so the result follows immediately.

Necessity. If $B$ is not torsion free, then we have a submodule of the form $R / \mathfrak{m}$ with $\mathfrak{m}$ maximal, and if $B$ is not reduced, then it contains a submodule isomorphic to the quotient field $Q$ of $R$, and neither of these is slender. Clearly $B$ is not slender if it contains a submodule isomorphic to $P$. Finally, suppose $B$ has an $\mathscr{C}$-adically complete submodule, and let $\left\{b_{n}\right\}$ be a sequence of elements in this submodule which $\mathscr{C}$-adically converges to 0 . Then we define $\phi: P \rightarrow B$ by $\phi(x)=\sum_{1}^{\infty} x(i) b_{i}$, and $\phi$ fails to be continuous.

I would like to express my thanks to my adviser, F. Richman, for his many helpful suggestions and for having helped to put all this into presentable form.

\section{REFERENCES}

1. H. Bass, Big projective modules are free, Illinois J. Math., 7 (1963), 24-31.

2. Bourbaki, Topologie Generale, Chapter IX.

3. L. Fuchs, Abelian Groups, Pergamon Press, New York.

4. Y. Hinohara, Projective modules over weakly Noetherian rings, J. Math. Soc. Japan, 15 (1963), 75-88 and 474-475.

5. R. Nunke, On direct products of infinite cyclic groups, Proc. Amer. Math. Soc., 13 (1962), 66-71.

6. - Slender groups, Acta Sci. Math. Szegd., 23 (1962), 67-73.

7. S. Ulam, Zur masstheorie in der allgemein mengenlehre, Fund. Math., 16 (1930), 140-150.

Received March 23, 1972.

New Mexico State University 


\section{PACIFIC JOURNAL OF MATHEMATICS}

\section{EDITORS}

RICHARD ARENS (Managing Editor)

University of California

Los Angeles, California 90024

\section{R. A. Beaumont}

University of Washington

Seattle, Washington 98105

\section{J. DuGundJI*}

Department of Mathematics

University of Southern California

Los Angeles, California 90007

D. Gilbarg and J. Milgram

Stanford University

Stanford, California 94305

ASSOCIATE EDITORS
E. F. BECKENBACH
B. H. NeumanN
F. WOLF
K. YoSHIDA

\section{SUPPORTING INSTITUTIONS}

UNIVERSITY OF BRITISH COLUMBIA

CALIFORNIA INSTITUTE OF TECHNOLOGY

UNIVERSITY OF CALIFORNIA

MONTANA STATE UNIVERSITY

UNIVERSITY OF NEVADA

NEW MEXICO STATE UNIVERSITY

OREGON STATE UNIVERSITY

UNIVERSITY OF OREGON

OSAKA UNIVERSITY

\author{
UNIVERSITY OF SOUTHERN CALIFORNIA \\ STANFORD UNIVERSITY \\ UNIVERSITY OF TOKYO \\ UNIVERSITY OF UTAH \\ WASHINGTON STATE UNIVERSITY \\ UNIVERSITY OF WASHINGTON \\ AMERICAN MATHEMATICAL SOCIETY \\ NAVAL WEAPONS CENTER
}

The Supporting Institutions listed above contribute to the cost of publication of this Journal, but they are not owners or publishers and have no responsibility for its content or policies.

Mathematical papers intended for publication in the Pacific Journal of Mathematics should be in typed form or offset-reproduced, (not dittoed), double spaced with large margins. Underline Greek letters in red, German in green, and script in blue. The first paragraph or two must be capable of being used separately as a synopsis of the entire paper. Items of the bibliography should not be cited there unless absolutely necessary, in which case they must be identified by author and Journal, rather than by item number. Manuscripts, in duplicate if possible, may be sent to any one of the four editors. Please classify according to the scheme of Math. Rev. Index to Vol. 39. All other communications to the editors should be addressed to the managing editor, or Elaine Barth, University of California, Los Angeles, California, 90024.

50 reprints are provided free for each article; additional copies may be obtained at cost in multiples of 50 .

The Pacific Journal of Mathematics is issued monthly as of January 1966. Regular subscription rate: $\$ 48.00$ a year (6 Vols., 12 issues). Special rate: $\$ 24.00$ a year to individual members of supporting institutions.

Subscriptions, orders for back numbers, and changes of address should be sent to Pacific Journal of Mathematics, 103 Highland Boulevard, Berkeley, California, 94708.

\section{PUBLISHED BY PACIFIC JOURNAL OF MATHEMATICS, A NON-PROFIT CORPORATION}

Printed at Kokusai Bunken Insatsusha (International Academic Printing Co., Ltd.), 270, 3-chome Totsuka-cho, Shinjuku-ku, Tokyo 160, Japan.

* C. R. DePrima California Institute of Technology, Pasadena, CA 91109, will replace J. Dugundji until August 1974.

Copyright (C) 1973 by

Pacific Journal of Mathematics

All Rights Reserved 


\section{Pacific Journal of Mathematics}

\section{Vol. 49, No. $2 \quad$ June, 1973}

Wm. R. Allaway, On finding the distribution function for an orthogonal polynomial

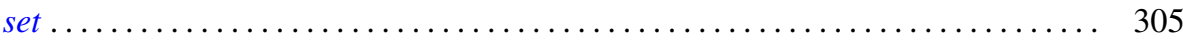

Eric Amar, Sur un théorème de Mooney relatif aux fonctions analytiques bornées... . 311

Robert Morgan Brooks, Analytic structure in the spectrum of a natural system . . . . 315

Bahattin Cengiz, On extremely regular function spaces . . . . . . . . . . . . . . 335

Kwang-nan Chow and Moses Glasner, Atoms on the Royden boundary . . . . . . . . . 339

Paul Frazier Duvall, Jr. and Jim Maxwell, Tame $Z^{2}$-actions on $E^{n} \ldots \ldots \ldots \ldots \ldots . .349$

Allen Roy Freedman, On the additivity theorem for $n$-dimensional asymptotic

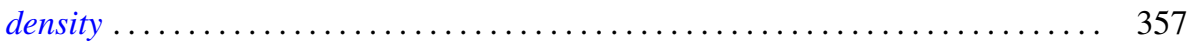

John Griffin and Kelly Denis McKennon, Multipliers and the group $L_{p}$-algebras . . . 365

Charles Lemuel Hagopian, Characterizations of $\lambda$ connected plane continua ....... 371

Jon Craig Helton, Bounds for products of interval functions . . . . . . . . . . . 377

Ikuko Kayashima, On relations between Nörlund and Riesz means . . . . . . . . . . 391

Everett Lee Lady, Slender rings and modules . . . . . . . . . . . . . . . . . . 397

Shozo Matsuura, On the Lu Qi-Keng conjecture and the Bergman representative

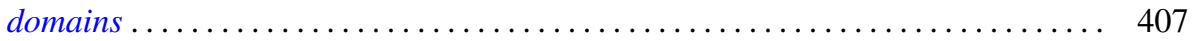

Stephen H. McCleary, The lattice-ordered group of automorphisms of an $\alpha$-set . . . 417

Stephen H. McCleary, o-2-transitive ordered permutation groups .......... 425

Stephen H. McCleary, o-primitive ordered permutation groups. II . . . . . . . . . 431

Richard Rochberg, Almost isometries of Banach spaces and moduli of planar

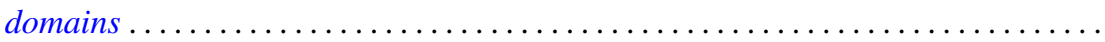

R. F. Rossa, Radical properties involving one-sided ideals . . . . . . . . . . . . . 467

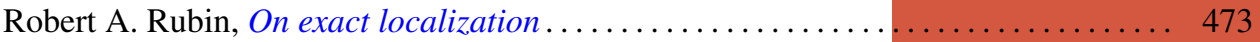

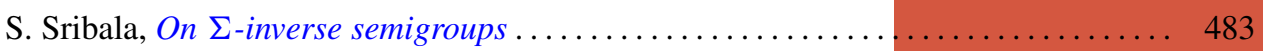

H. M. (Hari Mohan) Srivastava, On the Konhauser sets of biorthogonal polynomials suggested by the Laguerre polynomials ...................... 489

Stuart A. Steinberg, Rings of quotients of rings without nilpotent elements . ...... 493

Daniel Mullane Sunday, The self-equivalences of an $H$-space . . ............ 507

W. J. Thron and Richard Hawks Warren, On the lattice of proximities of $\check{C} e c h$

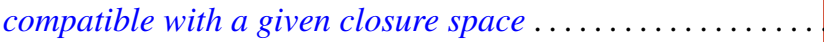

Frank Uhlig, The number of vectors jointly annihilated by two real quadratic forms determines the inertia of matrices in the associated pencil .

Frank Uhlig, On the maximal number of linearly independent real vectors annihilated simultaneously by two real quadratic forms ..............

Frank Uhlig, Definite and semidefinite matrices in a real symmetric matrix pencil . . 561

Arnold Lewis Villone, Self-adjoint extensions of symmetric differential operators . . . 569

Cary Webb, Tensor and direct products . ....................... 579

James Victor Whittaker, On normal subgroups of differentiable

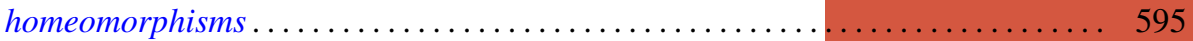

Jerome L. Paul, Addendum to: "Sequences of homeomorphisms which converge to

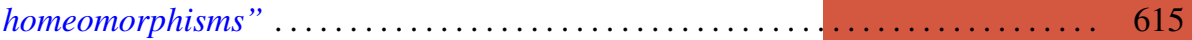

David E. Fields, Correction to: “Dimension theory in power series rings” ........ 616

Peter Michael Curran, Correction to: “Cohomology of finitely presented groups”. . . 617

Billy E. Rhoades, Correction to: “Commutants of some Hausdorff matrices” ...... 617

Charles W. Trigg, Corrections to: "Versum sequences in the binary system” ...... 619 